\title{
El impacto que tiene el internet en los procesos educativos inclusivos desde la perspectiva del docente
}

The impact of the internet in the inclusive educational processes from the perspective of the teacher

0 impacto da internet nos processos educacionais inclusivos na perspectiva do profesor

Yuli Muñoz'

Corporación Universitaria Iberoamericana (Colombia)

Luz Rojas-Morales

Corporación Universitaria Iberoamericana (Colombia)

Luz Mosquera-Lozano

Corporación Universitaria Iberoamericana (Colombia)

DOI: https://doi.org/10.35622/j.rep.2021.02.002

Recibido 02/12/2020/ Aceptado 20/03/2021

RESUMEN. El objetivo principal de esta investigación fue identificar el impacto que tiene el Internet en los procesos educativos inclusivos desde la perspectiva del docente de la Institución Educativa (I.E.) Nuestra Señora de La Macarena Meta en el año 2020. La metodología se basó en el enfoque cualitativo y diseño fenomenológico. La población definida estuvo conformada por 54 docentes de aula de la I.E. Nuestra Señora de La Macarena Meta, con edades entre los 26 y 65 años, utilizando como técnica e instrumento de recolección de información la aplicación de una entrevista semiestructurada compuesta por siete preguntas abiertas, a través de la cual se pretendió indagar sobre el impacto que tiene el Internet en los procesos educativos inclusivos desde la perspectiva del docente. Los resultados obtenidos de manera global reflejan que el municipio de La Macarena presenta deficiente conectividad de Internet, lo que no ha favorecido totalmente el uso de este en los procesos educativos inclusivos. Concluyendo finalmente, que el impacto que tiene el Internet en los procesos educativos inclusivos desde la perspectiva del docente fue positivo, debido a que, a pesar de las dificultades de conexión, permitió darles continuidad a dichos procesos en la institución educativa.

PALABRAS CLAVE: Internet, procesos educativos inclusivos, calidad, perspectiva, docente

ABSTRACT. The main objective of this research was to identify the impact that the Internet has on inclusive educational processes from the perspective of the teacher of the Educational Institution (IE) Nuestra Señora de La Macarena Meta in 2020. The methodology was based on a qualitative approach and phenomenological design. The defined population consisted of 54 classroom teachers from the I.E. Our Lady of La Macarena Meta, aged between 26 and 65, using the application of a semi-structured interview composed of seven open questions as a technique and instrument for collecting information, through which it was intended to inquire about the impact it has the Internet in inclusive educational processes from the teacher's perspective. The results obtained globally reflect that the municipality of La Macarena has poor Internet connectivity, which has not fully favored its use in inclusive educational processes. Finally, concluding that the impact that the Internet has on inclusive educational processes from the teacher's perspective was positive, because, despite the difficulties of connection, it allowed continuity of said processes in the educational institution.

${ }^{1}$ Correspondencia: yuli.munoz2804@gmail.com 
KEYWORDS: Internet, inclusive educational processes, quality, perspective, teacher.

RESUMO. 0 objetivo principal desta pesquisa foi identificar 0 impacto que a Internet tem nos processos educacionais inclusivos na perspectiva da professora da Instituição de Ensino (IE) Nuestra Señora de La Macarena Meta em 2020. A metodologia foi baseada em uma abordagem qualitativa e design fenomenológico. A população definida foi composta por 54 professores do I.E. Nossa Senhora da Macarena Meta, com idade entre 26 e 65 anos, utilizando como técnica e instrumento de recolha de informação a entrevista semiestruturada composta por sete questões abertas, através da qual se pretendeu indagar sobre o impacto que tem na Internet em processos educacionais inclusivos na perspectiva do professor. Os resultados obtidos globalmente refletem que o município de La Macarena possui baixa conectividade à Internet, o que não favoreceu totalmente seu uso em processos educacionais inclusivos. Por fim, concluindo que o impacto que a Internet tem nos processos educacionais inclusivos na perspectiva do professor foi positivo, pois, apesar das dificuldades de conexão, permitiu a continuidade desses processos na instituição educacional.

PALABRAS CLAVE: Internet, processos educacionais inclusivos, qualidade, perspectiva, professor.

\section{INTRODUCCIÓN}

La educación inclusiva es un eje fundamental para el desarrollo y formación integral de los niños, niñas, adolescentes y jóvenes (NNAJ), la cual debe ofrecer entornos adecuados y adaptados a la población estudiantil de acuerdo a cada una de las características, capacidades, intereses, necesidades, particularidades y diversidad de los NNAJ, para que los procesos de enseñanza y aprendizaje garanticen y permitan el goce efectivo del Derecho a la educación, como lo establece la Ley 1098 de 2006 en su Artículo 28 "Ios niños, las niñas y los adolescentes tienen derecho a una educación de calidad". Igualmente, la UNESCO (2009), desde los derechos fundamentales, define la educación inclusiva como el derecho de todos los estudiantes a recibir una educación de calidad que satisfaga sus necesidades básicas de aprendizaje y enriquezca sus vidas.

La educación inclusiva, más que un tema marginal que trata sobre cómo integrar a ciertos estudiantes a la enseñanza convencional, representa una perspectiva que debe servir para analizar cómo transformar los sistemas educativos y otros entornos de aprendizaje, con el fin de responder a la diversidad de los estudiantes. El propósito de la educación inclusiva es permitir que los maestros y estudiantes se sientan cómodos ante la diversidad y la perciban no como un problema, sino como un desafío y una oportunidad para enriquecer las formas de enseñar y aprender (UNESCO, 2005, p.14).

Según Ainscow y Echeita (2011) resaltan cuatro elementos de la educación inclusiva: la inclusión es un proceso; busca la presencia, la participación y el éxito de todos los estudiantes; la inclusión precisa la identificación y la eliminación de barreras y la inclusión pone particular énfasis en aquellos grupos de alumnos que podrían estar en riesgo de marginalización, exclusión, o fracaso escolar.

Al realizar una lectura del contexto para Colombia, se encontró según el Consejo Nacional de Política Económica y Social (CONPES, 2020), que existe una deficiencia de conectividad a Internet en las sedes educativas, lo cual aumentó debido a que por la situación de la 
emergencia sanitaria. Las I.E. debieron recurrir a una educación virtual a través del Internet para continuar con la educación, evidenciando el aumento de los problemas de conectividad, teniendo el mayor impacto en los procesos de educación inclusiva.

Es así, que el objetivo de esta investigación fue identificar el impacto que tiene el Internet en los procesos educativos inclusivos desde la perspectiva de los docentes de la Institución Educativa Nuestra Señora de La Macarena Meta, en el año 2020.

Partiendo del objetivo, se abrieron caminos para el desarrollo de esta investigación, donde se tiene en cuenta el Internet como un elemento necesario y significativo en los procesos de enseñanza y aprendizaje, en ese sentido, según Castells (2015), considera el internet como una de las características más significativas del tiempo; además de ser un poderoso instrumento para el proceso de la información, facilitar el aprendizaje mediante el uso de materiales didácticos interactivos; añade la posibilidad de acceder a todo tipo de información y sobre todo, abre un canal. Con ello, se abre un nuevo paradigma de la enseñanza, sin barreras espacio temporal para el acceso a la información y para la comunicación interpersonal, ofreciendo múltiples posibilidades de innovación educativa en el marco de la enseñanza más personalizada y de un aprendizaje cooperativo acorde con los planteamientos socio constructivistas.

Las Tecnologías de la Información y Comunicaciones - TIC son herramientas que promueven factores de equidad, pertinencia e inclusión educativa en los ambientes de aprendizaje escolares y favorecen el desarrollo de habilidades como razonar, inferir, interpretar, argumentar, proponer, modelar, tomar decisiones, resolver problemas, entre otros. Por tanto, su uso como mediadores del aprendizaje, incluso en personas en situación de discapacidad, es fundamental para alcanzar el propósito de calidad educativa (Watts \& Lee, 2017).

Existen varias investigaciones que se relacionaron con el objeto de estudio, dentro de las cuales se encuentra la investigación realizada por Mejía y Gómez (2017), acerca del Internet como herramienta didáctica, cuyo objetivo principal fue analizar el uso del Internet como herramienta didáctica en el proceso de aprendizaje educativo en los alumnos del nivel medio superior de la preparatoria 14 de la Universidad Autónoma de Nayarit México (UAN), a partir del enfoque cuantitativo y diseño no experimental de alcance descriptivo, encontrando como resultados que la importancia de contar con Internet en el hogar es prioritaria para el desarrollo de tareas y consulta de la información, dado que aquellos que no cuentan con este tipo de herramientas les impide el acceso a las mismas, planteando que Internet ha dado inicio a desarrollar nuevos modelos y estrategias de aprendizaje, para fortalecer la formación de los estudiantes.

Watts y Lee (2017), hablan de las TIC como herramientas de inclusión educativa, que tuvo como objetivo identificar los diferentes referentes teóricos y características de la inclusión, así como reconocer el rol de las TIC como herramientas para la inclusión educativa y la generación de ambientes de aprendizaje participativos, desde un trabajo monográfico, el 
cual arrojó como resultados que las posibilidades de las TIC son diversas, pero al mismo tiempo limitadas; debido a que la mayoría de sistemas, no permiten el acceso igual y equitativo para todos, lo que les permitió reconocer que el rol de las TIC en la práctica de la educación inclusiva es fundamental, dado que su utilización, por un lado, favorece el propósito de una educación de calidad, pero además, con su aplicación, se puede eliminar las barreras que impiden el acceso a la formación.

A partir de las investigaciones que se realizaron a nivel internacional, nacional y local, se pudo concluir que estas permitieron conocer aspectos relevantes que direccionaron el presente trabajo de investigación desarrollado, pues se evidenciaron en los resultados, argumentos suficientes que permitieron justificar la necesidad de determinar el impacto que tiene el Internet en los procesos educativos inclusivos desde la perspectiva del docente de la Institución Educativa Nuestra Señora de La Macarena Meta en el año 2020, pues para nadie es un secreto que la situación actual de la emergencia sanitaria en el mundo y en nuestro país, nos ha llevado a cambiar no solo nuestros estilos de vida, sino también la educación y parte de estos cambios tienen que ver con el Internet para poder llevar la educación a cada uno de los estudiantes, y que esta nueva estrategia de educación tenga las herramientas suficientes para brindar unos procesos educativos inclusivos.

\section{METODOLOGÍA}

\section{Diseño}

El proyecto de investigación se realizó desde el enfoque cualitativo, ya que este "se enfoca en comprender los fenómenos, explorándolos desde la perspectiva de los participantes en un ambiente natural y en relación con su contexto" (Hernández, Fernández y Baptista, 2014, p.358). Es así, como este estudio estuvo orientado en identificar el impacto que tiene el Internet en los procesos educativos inclusivos desde la perspectiva del docente de la Institución Educativa Nuestra Señora de La Macarena Meta en el año 2020, teniendo en cuenta, el análisis de su percepción y experiencia de este fenómeno, tomando sus puntos de vista, interpretaciones y significados dados a la educación inclusiva.

Se utilizó el diseño fenomenológico, que tiene como finalidad explicar la naturaleza de las cosas, la esencia y la veracidad de los fenómenos, desde la perspectiva de los participantes en los procesos de investigación, para comprender las experiencias vividas en su complejidad, comprender el fenómeno con una mirada holística, como parte de un todo significativo y toma de conciencia en cuanto al suceso (Husserl, 1998). Por esta razón, se consideró este diseño como un elemento muy apropiado y pertinente para el desarrollo de la investigación, dado que la perspectiva del docente juega un papel importante, necesario y significativo en el tema énfasis de este estudio investigativo. 
Población - Muestra

La muestra es igual a la población objeto de investigación, estuvo conformada por 54 docentes de aula de la I.E. Nuestra Señora de La Macarena Meta, con edades entre los 26 y 65 años, género femenino 35 y masculino 19, tiempo laborado en la I.E. entre 7 meses y 29 años, los cuales cuentan con un nivel académico desde normalista superior hasta magister.

De los cuales, 48 docentes pertenecen a la zona urbana y 6 a la zona rural, distribuidos por cada una de las sedes de esta manera: en la cabecera municipal 27 en la sede principal Nuestra Señora de la Macarena y 21 en la Escuela John F. Kennedy y uno por cada sede rural (El Billar, Bajo Raudal, Alto Raudal, El Tapir y Juan León), excepto la sede Juan León que cuenta con dos docentes.

Técnicas de Recolección de Información

La Entrevista.

Es una técnica de recolección de información que permite indagar sobre los aspectos presentes en el contexto a investigar, como según Bautista (2011), la define en una técnica de investigación que permite obtener datos mediante un diálogo entre el investigador y el entrevistado, como una conversación con una Para este trabajo se eligió la entrevista semiestructurada en la cual "el investigador dispone de una serie de temas que debe trabajar a lo largo de la entrevista, pero puede decidir libremente sobre el orden de presentación de los diversos temas y el modo de formular la pregunta" (Corbetta, 2007, como se citó en Batthyány y Cabrera, 2011, p.90). Por esta razón, se consideró que este tipo de entrevista es pertinente para el desarrollo de esta investigación, dado que permite al investigador obtener ampliación de la información y mayor acercamiento a la población objeto, y al entrevistado tener libertad de expresión, sin restricciones para dar sus puntos de vista del contexto abordado, ofreciendo así un espacio flexible y activo de participación para las partes.

Instrumentos.

Para el objeto de esta investigación se utilizó la entrevista semiestructura, con el fin de ser aplicada de manera presencial y virtual a los docentes de aula, permitiendo tener un acercamiento y participación con uno de los actores principales en los procesos educativos inclusivos de la I.E. Nuestra Señora de la Macarena Meta, compuesta por título, objetivo, concepto de procesos educativos inclusivos, dimensiones y características de la educación inclusiva, datos personales y siete preguntas abiertas a través de la cual se pretendió indagar sobre el impacto que tiene el Internet en los procesos educativos inclusivos desde la perspectiva del docente. 


\section{Consideraciones Éticas}

El desarrollo de este trabajo de investigación se rigió basado en las consideraciones éticas establecidas en la Resolución 008430 de 1993 definida por el Ministerio de Salud de Colombia, con el fin de proteger a la población sujeto de investigación, para lo cual se tuvo en cuenta los Artículos 5 en el que debe prevalecer el respeto a la dignidad y la protección de los derechos y bienestar del sujeto de estudio; Artículo 6 puntualizando en los criterios: a) ajustado a los principios científicos y éticos, d) Debe prevalecer la seguridad de los beneficiarios y expresar claramente los riesgos (mínimos) que deben estar en concordancia con el Artículo 11 de esta resolución y e) Contar con el consentimiento informado del sujeto de investigación; Artículo 8 busca proteger la privacidad de la persona y finalmente, el Artículo 11 que para efectos de la investigación se tomó de este, el ítem a)Investigación sin riesgo, porque se acoge pertinentemente a este proceso.

\section{Procedimientos}

El trabajo desarrollado se dividió en tres fases, descritas así: en la fase 1, se realizó el acercamiento de manera presencial y virtual a la Institución Educativa Nuestra Señora de La Macarena, analizando desde la situación surgida por la emergencia sanitaria que se presentó en el año 2020, el aumento de los problemas de conexión a Internet y el impacto que esto tiene en los procesos de educación inclusiva, evidenciado en lo observado y vivido desde la experiencia laboral, estudiantil y familiar de esta institución. Detectada la necesidad, se decidió abordarla desde la perspectiva de los docentes como uno de los principales actores en los procesos educativos inclusivos y para esto se propuso trabajar con la totalidad de los 54 docentes activos de la I.E. en el año 2020.

La I.E. dio a conocer la caracterización de los docentes, exponiendo que la población se encuentra distribuida en 7 sedes educativas, 2 ubicadas en la zona urbana y 5 en la zona rural, con perfiles desde normalistas superior hasta magister y edades entre los 26 y 65 años.

Se formularon los objetivos a alcanzar con la población seleccionada para el desarrollo de este trabajo investigativo; seguido por la elaboración del instrumento "Formato de entrevista semiestructurada", que va en función de identificar el impacto que tiene el Internet en los procesos educativos inclusivos desde la perspectiva del docente.

En la fase 2, se aplicó el instrumento "Formato de entrevista semiestructurada" orientado desde un preámbulo de los conceptos teóricos de los procesos educativos inclusivos en los cuales se basó esta investigación, buscando dar respuesta a los tres objetivos específicos: 1) establecer las formas de acceso a Internet que tienen los estudiantes; 2) examinar la calidad de los procesos educativos inclusivos desde la perspectiva del docente y 3) analizar el impacto que tiene el Internet en los procesos educativos inclusivos. Para alcanzar el objetivo general a través de la formulación de siete preguntas abiertas, brindando a los docentes un espacio participativo de libre expresión frente a sus conocimientos, 
experiencias educativas vividas y evidenciadas en el año mencionado anteriormente, como también la oportunidad de que realizaran un aporte de aquellas acciones que desde el uso del Internet se puedan implementar para favorecer los procesos educativos inclusivos de la I.E.

Y en la fase 3, se organizó la información recopilada durante el proceso de investigación con el propósito de tabular, sistematizar, analizar, obtener resultados y concluir. Una vez la información se sistematizó, se produjeron los hallazgos y las conclusiones, se procedió a socializar el trabajo realizado y consolidado con la Corporación Universitaria Iberoamericana y con la Institución Educativa Nuestra Señora de La Macarena que abrió las puertas para la realización del trabajo investigativo.

\section{RESULTADOS}

\section{Codificación abierta}

Tabla 1. Análisis de resultados por subcategorías codificación abierta.

\begin{tabular}{|c|c|}
\hline CÓDIGOS DE SUBCATEGORÍAS & TOTAL CÓDIGOS \\
\hline (CFCE)En casa de un familiar o compañero de estudio & 30 \\
\hline (SAI)Sin acceso a Internet & 29 \\
\hline (DMPR)Datos móviles prepago & 25 \\
\hline (WC)Wifi en casa & 21 \\
\hline (DMPO)Datos móviles postpago & 19 \\
\hline (RP)Redes públicas & 18 \\
\hline$(\mathrm{ClHT})$ Compra de Internet por horas a terceros & 16 \\
\hline (DC)Datos compartidos & 15 \\
\hline (Cl)Café Internet & 11 \\
\hline (O)Otra & 1 \\
\hline (D)Desconoce & 0 \\
\hline Redes sociales, entretenimiento y otras actividades & 10 \\
\hline Actividades académicas & 9 \\
\hline Deficiente conectividad & 8 \\
\hline Sin Internet & 6 \\
\hline $\begin{array}{l}\text { Acompañamiento y apoyo de padres/madres de familia y } \\
\text { cuidadores }\end{array}$ & 2 \\
\hline Comunicación & 1 \\
\hline Facilita y fortalece la enseñanza y el aprendizaje & 18 \\
\hline $\begin{array}{l}\text { Herramienta útil, práctica y esencial para el desarrollo de } \\
\text { las actividades académicas }\end{array}$ & 10 \\
\hline Desigualdad de condiciones & 9 \\
\hline Sin Internet & 7 \\
\hline Comunicación & 4 \\
\hline Innovación & 4 \\
\hline Aprendizaje significativo & 3 \\
\hline
\end{tabular}


Cobertura educativa

3

Capacitación y formación

3

Deficiente conectividad

3

Aprovechamiento inadecuado

2

Desarrollo de nuevas capacidades e interés para la 2

búsqueda de información

Autoaprendizaje 1

Espacios nuevos de socialización, interacción e intercambio de ideas

Facilita y fortalece la enseñanza y el aprendizaje

22

Herramienta útil, práctica y esencial para el desarrollo de las actividades académicas

Comunicación

Desigualdad de condiciones

Aprendizajes prácticos y significativos

6

Sin Internet

5

Desarrollo de nuevas capacidades e interés para la búsqueda de información

Acompañamiento y apoyo de padres/madres de familia y cuidadores

Cobertura educativa 3

Resultados 3

Innovación 2

Deficiente conectividad 1

Continuidad de los procesos académicos 1

Autoaprendizaje 1

\begin{tabular}{ll}
\hline Enseñanza y el aprendizaje & 17
\end{tabular}

Aportes materiales y humanos $\quad 8$

Resultados

Características del educando 4

Escasez de Aportes materiales $\quad 4$

Aprendizajes prácticos y significativos 4

Innovación 4

Capacitación y formación 4

Acompañamiento y apoyo de padres/madres de familia y 4

cuidadores

Sin Internet 4

Contextos 3

Comunicación 1

Continuidad de los procesos académicos 1

Continuidad de los procesos académicos 11

Enseñanza y el aprendizaje $\quad 10$

$\begin{array}{ll}\text { Aportes materiales y humanos } & 7\end{array}$

Capacitación y formación $\quad 7$

Deficiente conectividad 6

Contextos 5

Resultados

Yuli Muñoz; Luz Rojas-Morales; Luz Mosquera-Lozano 
Aprendizajes prácticos y significativos

Sin Internet

Desigualdad de condiciones

Comunicación

Características del educando

Innovación

Acompañamiento y apoyo de padres/madres de familia y 2

cuidadores

Autoaprendizaje 2

Mejoramiento continuo

Enseñanza y el aprendizaje 11

Nuevos recursos materiales $\quad 11$

Mejoramiento continuo 11

Actualización e innovación $\quad 10$

Desigualdad de condiciones 10

Capacitación y formación $\quad 10$

Sin Internet 9

Resultados $\quad 7$

Deficiente conectividad 5

Contextos 3

Continuidad de los procesos académicos 3

Aprendizaje significativo 2

Espacios nuevos de socialización e interacción 2

Pensamiento crítico 1

Autonomía 1

Fuente: Elaboración propia de los datos obtenidos en las entrevistas a docentes.

\section{Codificación axial}

Tabla 2. Análisis de resultados por categorías codificación axial.

\begin{tabular}{|c|c|c|}
\hline CATEGORÍAS & CÓDIGOS DE SUBCATEGORÍAS & $\begin{array}{l}\text { TOTAL } \\
\text { CÓDIGOS }\end{array}$ \\
\hline \multirow{11}{*}{$\begin{array}{l}\text { Formas de Acceso a } \\
\text { Internet }\end{array}$} & $\begin{array}{l}\text { (CFCE)En casa de un familiar o compañero } \\
\text { de estudio }\end{array}$ & 30 \\
\hline & (SAI)Sin acceso a Internet & 29 \\
\hline & (DMPR)Datos móviles prepago & 25 \\
\hline & (WC)Wifi en casa & 21 \\
\hline & (DMPO)Datos móviles postpago & 19 \\
\hline & (RP)Redes públicas & 18 \\
\hline & $\begin{array}{l}\text { (CIHT)Compra de Internet por horas a } \\
\text { terceros }\end{array}$ & 16 \\
\hline & (DC)Datos compartidos & 15 \\
\hline & (Cl)Café Internet & 11 \\
\hline & (O)Otra & 1 \\
\hline & (D)Desconoce & 0 \\
\hline Uso del Internet & $\begin{array}{l}\text { Redes sociales, entretenimiento y otras } \\
\text { actividades }\end{array}$ & 10 \\
\hline
\end{tabular}

Yuli Muñoz; Luz Rojas-Morales; Luz Mosquera-Lozano 
Actividades académicas
Deficiente conectividad

9

Sin Internet

Acompañamiento y apoyo de padres/madres de familia y cuidadores

Comunicación

Facilita y fortalece la enseñanza y el

aprendizaje

Herramienta útil, práctica y esencial para el desarrollo de las actividades académicas

Desigualdad de condiciones

Sin Internet

Comunicación

Innovación

Influencia del Internet en los

Procesos Educativos Inclusivos

6

2

1

4

4

3

3
Aprent

Cobertura educativa

Capacitación y formación

Deficiente conectividad

Aprovechamiento inadecuado

Desarrollo de nuevas capacidades e interés para la búsqueda de información

Autoaprendizaje

Espacios nuevos de socialización, interacción e intercambio de ideas
8

3

3

2

2

1

1

Facilita y fortalece la enseñanza y el

aprendizaje

Herramienta útil, práctica y esencial para el desarrollo de las actividades académicas

Comunicación

Desigualdad de condiciones

Aprendizajes prácticos y significativos 5

\section{Estrategias Pedagógicas desde el Internet en los Procesos Educativos Inclusivos}

Sin Internet

Desarrollo de nuevas capacidades e interés para la búsqueda de información

Acompañamiento y apoyo de padres/madres de familia y cuidadores

Cobertura educativa 3

Resultados

Innovación

Deficiente conectividad

Continuidad de los procesos académicos

Autoaprendizaje

Enseñanza y el aprendizaje

1

Aportes materiales y humanos

17

Calidad de los Procesos

Resultados

8

Características del educando 
Escasez de Aportes materiales

4

Aprendizajes prácticos y significativos

4

Innovación

4

Capacitación y formación

4

Acompañamiento y apoyo de padres/madres

de familia y cuidadores

Sin Internet

Contextos

Comunicación

Continuidad de los procesos académicos

Continuidad de los procesos académicos

Enseñanza y el aprendizaje

Aportes materiales y humanos

Capacitación y formación

Deficiente conectividad

Contextos

Resultados

Impacto del Internet en los

Procesos Educativos Inclusivos

\section{Implementación de Nuevas Acciones desde el Uso de Internet en los Procesos Educativos Inclusivos}

Aprendizajes prácticos y significativos 4

Sin Internet 4

Desigualdad de condiciones 4

Comunicación 3

Características del educando 2

Innovación 2

Acompañamiento y apoyo de padres/madres 2

de familia y cuidadores

Autoaprendizaje 2

Mejoramiento continuo 1

Enseñanza y el aprendizaje 11

Nuevos recursos materiales $\quad 11$

Mejoramiento continuo 11

Actualización e innovación 10

Desigualdad de condiciones $\quad 10$

Capacitación y formación 10

Sin Internet 9

Resultados $\quad 7$

Deficiente conectividad 5

Contextos 3

Continuidad de los procesos académicos 3

Aprendizaje significativo 2

Espacios nuevos de socialización e interacción 2

Pensamiento crítico 1

Autonomía 1

Fuente: Elaboración propia de los datos obtenidos en las entrevistas a docentes 


\section{Codificación Selectiva}

A partir de la implementación del instrumento "Formato de entrevista semiestructurada" aplicado a 31 docentes de aula, con edades entre los 26 y 65 años, género femenino 21 y masculino 10, que para este análisis serán identificados con la letra "S" de sujeto y con un número de acuerdo al orden en que fueron entrevistados, se logró obtener los siguientes resultados que van en función de identificar el impacto que tiene el Internet en los procesos educativos inclusivos desde la perspectiva del docente de la Institución Educativa Nuestra Señora de La Macarena Meta en el año 2020, descritos en las categorías presentadas a continuación:

Formas de Acceso a Internet.

Tabla 3. Formas de acceso a Internet de los estudiantes de la IENSM en el año 2020

\begin{tabular}{cc} 
CRITERIO & TOTAL \\
\hline CFCE & 30 \\
SAI & 29 \\
DMPR & 25 \\
WC & 21 \\
DMPO & 19 \\
RP & 18 \\
CIHT & 16 \\
DC & 15 \\
Cl & 11 \\
O & 1 \\
D & 0
\end{tabular}

Fuente: $\overline{\text { Elaboración propia de los datos obtenidos en las entrevistas }}$ a docentes.

Figura 2. Formas de acceso a Internet de los estudiantes de la IENSM en el año 2020.

\section{FORMAS DE ACCESO A INTERNET DE LOS ESTUDIANTES DE LA IENSM EN EL AÑO 2020}

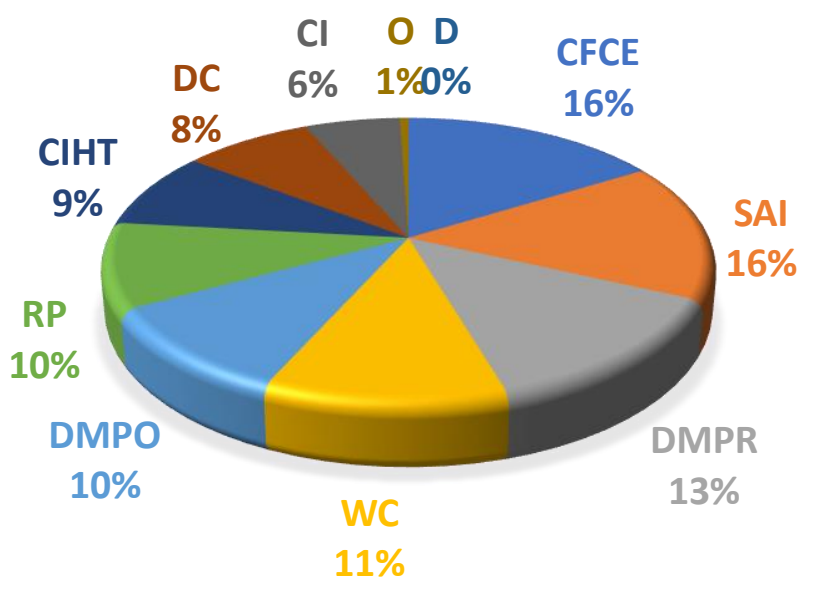

Fuente: Elaboración propia de los datos obtenidos en las entrevistas a docentes 
En la categoría "Formas de Acceso a Internet" se logró identificar desde el quehacer de los docentes las formas de acceso a Internet que tuvieron los estudiantes de la Institución Educativa Nuestra Señora de La Macarena Meta, mostrados de acuerdo a las respuestas dadas en los criterios de formas de acceso a Internet, así; en términos generales, se identificó que la forma de acceso a Internet (CFCE) en casa de un familiar o compañero de estudio es la que predomina obteniendo el 16\%, seguido en un punto intermedio por (RP) Redes públicas con 10\% y la de menor (O) Otra con el 1\%. Igualmente, se encuentra en esta categoría un porcentaje representativo del 16\% para el criterio (SAI) sin acceso a Internet. De esta manera, se establecieron las formas de acceso a Internet que tuvieron los estudiantes, analizando según lo expresado por los docentes y como los resultados lo muestran, que a pesar de la situación de emergencia sanitaria presentada en el año 2020 y de las dificultades de conectividad existentes en el municipio de La Macarena, los estudiantes buscaron y tuvieron diversas formas de acceso a Internet que les permitió continuar con su proceso académico desde la estrategia "Aprender en casa".

\section{Uso del Internet}

Tabla 4. Uso del Internet de los estudiantes en el desarrollo de las actividades académicas de la IENSM en el año 2020.

\begin{tabular}{cc} 
CRITERIO & TOTAL \\
\hline Bueno & 6 \\
Regular & 20 \\
Malo & 8 \\
No usa Internet & 1 \\
No tiene Internet & 0 \\
\hline
\end{tabular}

Fuente: Elaboración propia de los datos obtenidos en las entrevistas $a$ docentes.

Figura 3. Uso del Internet de los estudiantes en el desarrollo de las actividades académicas de la IENSM en el año 2020.

\section{USO DEL INTERNET DE LOS ESTUDIANTES EN \\ EL DESARROLLO DE LAS ACTIVIDADES \\ ACADÉMICAS DE LA IENSM EN EL AÑO 2020}

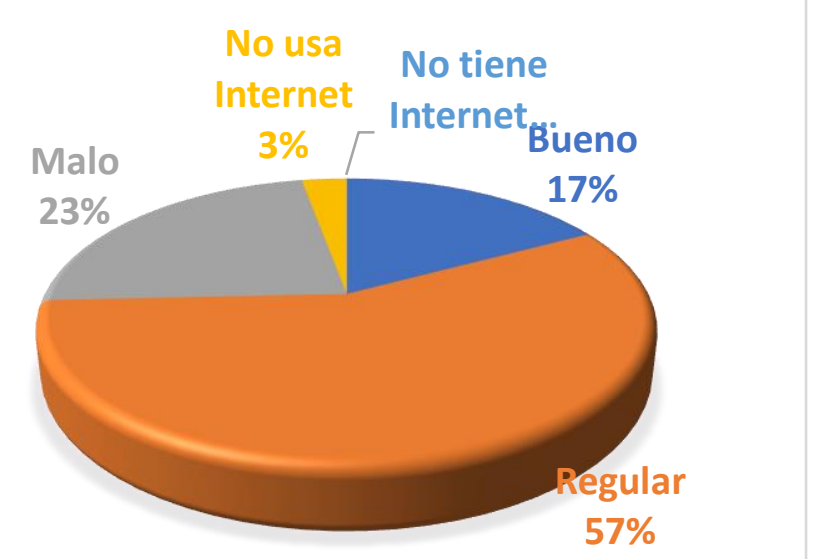

Fuente: Elaboración propia de los datos obtenidos en las entrevistas a docentes. 
En la categoría "Uso del Internet" se logró evidenciar desde la opinión de los docentes cómo fue el uso de Internet de los estudiantes en el desarrollo de las actividades académicas de la I.E. Nuestra Señora de la Macarena Meta en el año 2020, mostrados de acuerdo a las respuestas dadas en los criterios de uso del Internet, así: "Bueno" con un total de 6 respuestas correspondientes al 17\%, "Regular" con 20 representadas en el 57\%, "Malo" con 8 que corresponde al 23\%, "No usa Internet" con 1 correspondiente al 3\% y "No tiene Internet" con el 0\%. A manera general, se determinó que el uso del Internet fue "Regular" porque obtuvo el mayor porcentaje, 57\% y un porcentaje menor para el criterio "No usa Internet" con el $1 \%$.

El uso del Internet de los estudiantes estuvo determinado mayormente hacia las Redes sociales, entretenimiento y otras actividades.

"Muchos estudiantes no lo utilizaron para los medios de estudios si no para estar en juegos en otras aplicaciones menos para lo que tenían que utilizarla" (S25. P2).

Y en el desarrollo de las Actividades académicas.

"Si utilizaron mucho el Internet para hacer consultas y para ampliar las actividades a realizar pues y de esa forma adquirir más conocimiento" (S5. P2).

Sin embargo, se encontró que a pesar de que este fue utilizado para Actividades académicas, se constató que el uso del Internet de los estudiantes en el desarrollo de las actividades académicas, fue regular, debido a que estuvo marcado más hacia las Redes sociales, entretenimiento y otras actividades y también por las dificultades de conectividad que tiene el municipio.

"Primero porque las condiciones de conectividad no son las mejores en el municipio y en segundo lugar porque hay muchos que cuentan con el servicio pero que no lo utilizan con fines académicos sino de entretenimiento" (S7. P2).

"El índice de estudiantes con acceso a Internet acá en el municipio es muy bajo" (S10. P2).

\section{Influencia del Internet en los Procesos Educativos Inclusivos}

En la categoría "Influencia del Internet en los Procesos Educativos Inclusivos" se logró analizar desde el punto de vista de los docentes la manera como ha influido el Internet en los procesos educativos inclusivos en la I.E. Nuestra Señora de La Macarena Meta, encontrando que su influencia se evidenció principalmente en que facilita y fortalece la enseñanza y el aprendizaje, en que fue una Herramienta útil, práctica y esencial para el desarrollo de las actividades académicas y que permitió la Comunicación entre todos los actores de la comunidad educativa. 
El internet fue el mecanismo más utilizado en la I.E. para atender a la estrategia "Aprender en casa" implementada por el Ministerio de Educación Nacional, que permitió darle continuidad a la educación de todos los NNAJ, no sólo facilitando sino además fortaleciendo los procesos educativos inclusivos, logrando que la enseñanza llegará hasta sus hogares, que obtuvieran un aprendizaje continuo, pero a la vez nuevo y sobretodo significativo.

"El uso de ese Internet ha sido bastante significativo ya que se nos facilita (...) buscar actividades que sean llamativas e innovadoras para los estudiantes y de esa forma poderlos orientar mejor con las actividades que deben realizar desde casa, donde son ellos los pioneros de su propio aprendizaje, de su propio autoaprendizaje" (S5. P3).

Convirtiéndose en una Herramienta útil, práctica y esencial para el desarrollo de las actividades académicas, ya que permitió brindarles a los estudiantes la atención, orientación, retroalimentación, envío y recepción de trabajos.

"El Internet ha sido [en] un 80\% la herramienta más utilizada que tuvimos y que nos permitió llegar a cada uno de los estudiantes. Considero que sin el Internet, malo, bueno, prestado, bueno, montado en un árbol, buscando la señal, fue [la] que nos permitió llegar a cada uno de los rincones de los estudiantes, a sus familias, poderles explicar los procesos y que ellos hayan recibido retroalimentaciones medianamente mientras podían, entonces considero que el Internet fue crucial, fue uno de los factores importantes y principales en este proceso 2020" (S18. P3).

Del mismo modo, fue la herramienta principal, necesaria, eficaz y efectiva que permitió la comunicación entre todos los actores de la comunidad educativa.

"Notable, casi en un $100 \%$ porque pues debido al Internet es que nos pudimos comunicar tanto como docentes con directivos, docentesestudiantes, docentes-padres de familia" (S1. P3).

Por otro lado, se observó que no se reflejó influencia significativa del Internet por la desigualdad de condiciones entre los estudiantes, ya que no todos gozaron de las mismas condiciones económicas, sociales, de conectividad y recursos tecnológicos debido a las brechas existentes en el municipio.

"El Internet se ha convertido en un elemento indispensable para poder prestar el servicio educativo en esta situación de virtualidad y de estudio en casa, ya que ha sido la forma de llegar a cada uno de los estudiantes para poder orientar sus procesos educativos, sin embargo desde el contexto de la Macarena se ven desventajas entre unos estudiantes a otros, por desigualdad de condiciones, por lo tanto considero que su influencia ha sido un poco negativa" (S31. P3).

No obstante, se encontró y lo reflejaron los resultados que pese a que no todos los estudiantes contaron con las mismas condiciones, el Internet tuvo una influencia muy significativa en los procesos educativos inclusivos en la I.E. Nuestra Señora de La Macarena Meta en el año 2020. 


\section{Estrategias Pedagógicas desde el Internet en los Procesos Educativos Inclusivos}

Tabla 5. Estrategias pedagógicas desde el Internet en los procesos educativos inclusivos de los estudiantes de la IENSM en el año 2020.

\begin{tabular}{cc} 
CRITERIO DE FAVORECIMIENTO & TOTAL \\
\hline Si & 26 \\
No & 5 \\
\hline
\end{tabular}

Fuente: Elaboración propia de los datos obtenidos en las entrevistas a docentes.

Figura 4. Estrategias pedagógicas desde el Internet en los procesos educativos inclusivos de los estudiantes de la IENSM en el año 2020.

\section{ESTRATEGIAS PEDAGÓGICAS DESDE \\ EL INTERNET EN LOS PROCESOS \\ EDUCATIVOS INCLUSIVOS DE LOS \\ ESTUDIANTES DE LA IENSM EN EL}

AÑO 2020

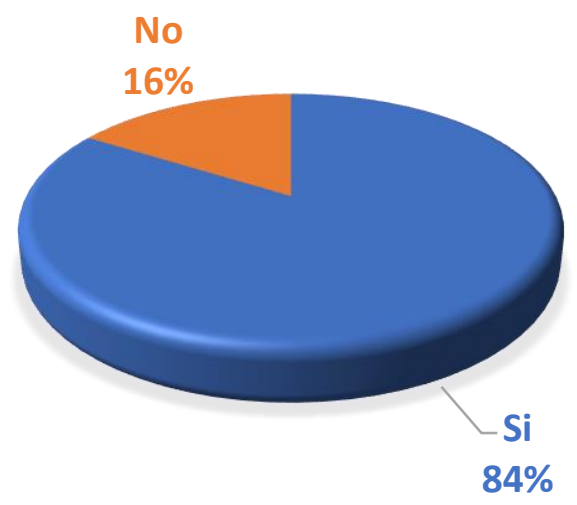

Fuente: Elaboración propia de los datos obtenidos en las entrevistas a docentes.

En la categoría "Estrategias Pedagógicas desde el Internet en los Procesos Educativos Inclusivos" se logró identificar desde la opinión de los docentes cuál fue el criterio de favorecimiento de las estrategias pedagógicas implementadas a través de Internet en los procesos educativos inclusivos de los estudiantes de la I.E. Nuestra Señora de la Macarena Meta, mostrados de acuerdo a las respuestas dadas en estos criterios, así: "Si" con un total de 26 respuestas representadas en el 84\% y 5 "No" con el 16\%. Es así, como los resultados detallaron que las estrategias pedagógicas desde el Internet "Si" favorecieron los Procesos Educativos Inclusivos reflejado en el porcentaje más alto del $84 \%$ y en un porcentaje menor "No" favorecieron con el $16 \%$.

De la misma manera, se evidenció que las Estrategias Pedagógicas desde el Internet en los Procesos Educativos Inclusivos, favorecieron las mismas subcategorías mencionadas en la anterior categoría, "Facilita y fortalece la enseñanza y el aprendizaje", "Herramienta útil, práctica y esencial para el desarrollo de las actividades académicas" y "Comunicación", resaltando dentro de estas las estrategias pedagógicas más sobresalientes implementadas 
por los docentes como: "video llamadas" (S14. P4), "los papás también participaran en el desarrollo de las guías virtualmente" (S15. P4), "grupos de WhatsApp" y "reuniones virtuales" (S16. P4), "clases grabadas" (S21. P4), "video" y "página para profundizar" (S23. P4) y "kioscos que se llaman vive digital" (S26. P4).

\section{Calidad de los Procesos Educativos Inclusivos}

Tabla 6. Calidad de los procesos educativos inclusivos en la IENSM en el año 2020.

\begin{tabular}{cc} 
CRITERIO & TOTAL \\
\hline Buena & 11 \\
Regular & 19 \\
Mala & 1 \\
Desconoce & 0 \\
\hline
\end{tabular}

Fuente: Elaboración propia de los datos obtenidos en las entrevistas $a$ docentes.

Figura 5. Calidad de los procesos educativos inclusivos en la IENSM en el año 2020.

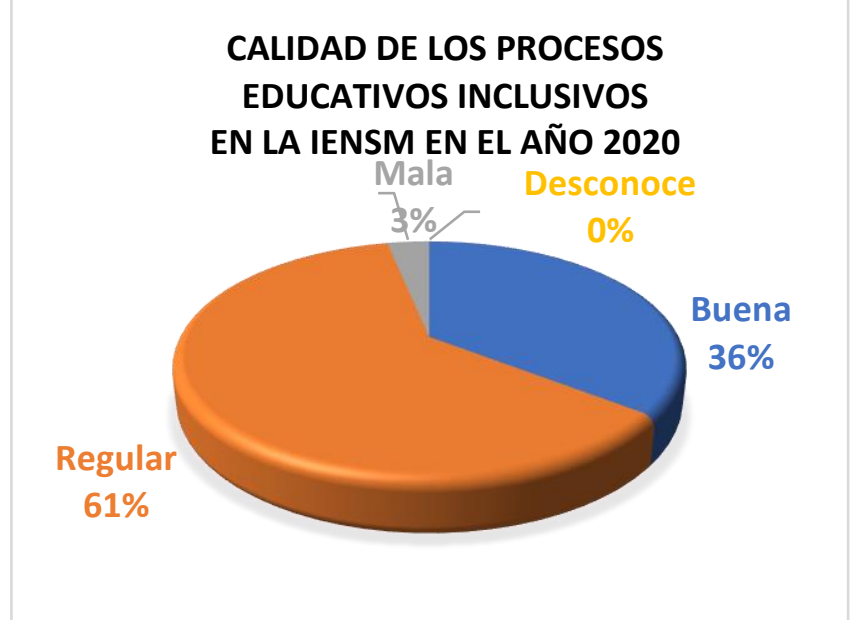

Fuente: Elaboración propia de los datos obtenidos en las entrevistas a docentes.

En la categoría "Calidad de los Procesos Educativos Inclusivos" se logró inferir desde el punto de vista de los docentes cómo fue la calidad de los procesos educativos inclusivos mostrados de acuerdo a las respuestas dadas en los criterios de calidad de los procesos educativos inclusivos, así: "Buena" con un total de 11 respuestas correspondientes al 36\%, "Regular" con 19 representadas en el 61\%, "Mala" con 1 que corresponde al 3\% y "Desconoce" con el $0 \%$. En general, se examinó que la calidad de los procesos educativos inclusivos fue "Regular" porque obtuvo el mayor porcentaje, $61 \%$, seguido en un punto intermedio por "Buena" con 36\% y el menor porcentaje "Mala" con el 3\%, teniendo en cuenta que para este análisis se tomó como referencia las cinco dimensiones propuestas por la UNESCO (2009), que tienen el objetivo de mejorar la calidad de la educación inclusiva, representadas como subcategorías, "Características del educando", "Contextos", "Aportes materiales y humanos", "Enseñanza y el aprendizaje" y "Resultados". 
Aunque la I.E. logró para el año 2020, continuar el proceso académico como se mencionó previamente, se observó que en los resultados en cuanto a la calidad de los procesos educativos inclusivos fueron determinantes las subcategorías, dado que, si bien la "Enseñanza y el aprendizaje1" se llevó a cabo, se desarrolló con algunas dificultades como la falta de acompañamiento y apoyo de padres/madres de familia y cuidadores, aprendizajes vacíos e incompletos, falta de capacitación en el uso de herramientas virtuales y dificultades de conectividad. Igualmente, en "Aportes materiales y humanos"2" se presentaron falencias como falta de herramientas y materiales físicos y tecnológicos. A pesar de que implementaron diferentes estrategias de enseñanza y aprendizaje, en "Resultados" no se alcanzaron los esperados. En "Características del educando", la I.E. realizó esfuerzos por atender a todos los estudiantes de acuerdo a sus condiciones y necesidades, sin embargo, tuvieron carencias como mayor capacitación para los docentes para que pudieran brindar una mejor atención a todos los estudiantes. Y en "Contextos", las limitaciones estuvieron dadas por fallas en la comunicación que conllevaron a que no pudieran llegar a la totalidad de los estudiantes, debido a la ubicación en zonas rurales dispersas y de difícil acceso. Todo lo anterior mencionado, fueron algunas de las razones principales por las cuales los docentes consideraron que la calidad fue regular.

"En el sentido que no es lo mismo enseñar desde casa, porque quedan muchos vacíos en cuanto al aprendizaje, la falta de tolerancia de los padres, del tiempo para dedicar a sus hijos y se evidenció que no alcanzaron el nivel que debían obtener de acuerdo a su nivel académico" (S30. P5).

"Regular, porque no se logra llegar al 100\% a cada uno de los estudiantes, en mi caso, para ser más precisa, hubo estudiantes con los que nunca tuve contacto directo, sino con terceras personas, entonces no podemos hablar de calidad en este caso" (S27. P5).

Contrario a los hallazgos anteriores, se encontró igualmente, según los resultados y lo expresado por los docentes que con todas las dificultades que tuvieron, la calidad de los procesos educativos inclusivas fue buena.

"Todos los estudiantes no tenían acceso de primera mano al Internet, sin embargo, considero que partimos también de las necesidades que tenían los estudiantes en casa y con base a sus necesidades y con base al uso del Internet, lo poquito que había, considero que las estrategias aplicadas fueron bastantes asertivas, bastantes puntualizadas y dieron el resultado que por ahora tenemos, que es poca deserción escolar" (S18. P5).

\section{Impacto del Internet en los Procesos Educativos Inclusivos}

Tabla 7. Impacto del Internet en los procesos educativos inclusivos en la IENSM en el año 2020.

\begin{tabular}{cc} 
CRITERIO & TOTAL \\
\hline Positivo & 16 \\
Regular & 12
\end{tabular}




$\begin{array}{cc}\text { Negativo } & 2 \\ \text { Ninguno } & 1 \\ \text { Desconoce } & 0\end{array}$

Fuente: Elaboración propia de los datos obtenidos en las entrevistas a docentes.

Figura 6. Impacto del Internet en los procesos educativos inclusivos en la IENSM en el año 2020.

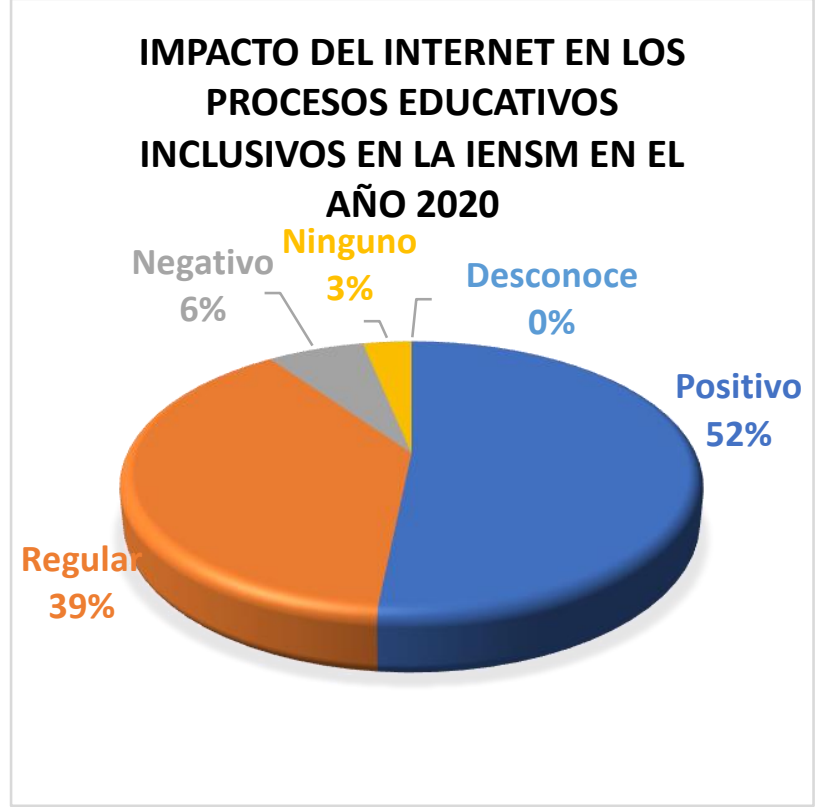

Fuente: Elaboración propia de los datos obtenidos en las entrevistas a docentes.

En la categoría "Impacto del Internet en los Procesos Educativos Inclusivos" se logró inferir desde la perspectiva de los docentes qué impacto tuvo el Internet en los procesos educativos inclusivos en la I.E., así: "Positivo" con un total de 16 respuestas correspondientes al 52\%, "Regular" con 12 representadas en el 39\%, "Negativo" con 2 que corresponde al 6\%, "Ninguno" con 1 representada en el 3\% y "Desconoce" con el 0\%. En términos generales, se analizó que el impacto que tuvo el Internet en los procesos educativos inclusivos fue "Positivo" porque obtuvo el mayor porcentaje, 52\%, seguido en un punto intermedio por "Regular" con 39\% y un porcentaje menor para el criterio "Ninguno" con el $3 \%$.

Desde lo positivo, en el año 2020, el Impacto del Internet en los Procesos Educativos Inclusivos en la I.E. estuvo determinado principalmente por la Continuidad de los procesos académicos, la Enseñanza y el aprendizaje, los Aportes materiales y humanos y la Comunicación, dado que permitió darle Continuidad a los procesos académicos inclusive abarcando a la gran mayoría de aquellos que viven en zonas retiradas, siendo el principal medio para el desarrollo, envió, recepción y evaluación de actividades.

"Ya que debido al Internet se suplieron muchas necesidades de estudio, muchos estudiantes pudieron continuar sus procesos académicos debido a que era la única manera de obtener su información. Por otro lado, si no hubiera existido el Internet, pues notablemente las guías no habían llegado 
con su totalidad a todos los estudiantes, porque hay unos que viven muy lejos, en zonas inhóspitas entonces es difícil” (S1. P6).

De igual manera, el impacto del Internet se vio reflejado en los procesos de Enseñanza y el aprendizaje, porque fue una ayuda elemental para que el trabajo académico se desarrollara, para la interacción y comunicación entre docentes, estudiantes y padres/madres de familia y cuidadores, que les brindó un espacio para aprender, investigar, consultar, tener la posibilidad de variedad de contenidos de acuerdo a las necesidades individuales, profundizar conceptos y conocimientos y adicional un espacio para relacionarse y conocer diferentes culturas, compartir e intercambiar ideas.

"La Internet nos ayuda a interactuar de manera impresionante, nos ayuda a acercarnos con diferentes culturas, diferentes conocimientos, diferentes ideas y el proceso educativo que ha tenido Nuestra Señora de La Macarena ha ayudado a que muchos estudiantes entiendan de que no solamente la Internet es sólo búsqueda en un sólo tipo de contenido, sino que esa si diversifica a las necesidades que ellos requirieron en su lugar de labor" (S4. P6).

En relación a los Aportes materiales y humanos, el impacto del Internet estuvo marcado porque posibilitó el acercamiento con los estudiantes a partir de diferentes herramientas tecnológicas y virtuales, las cuales ofrecieron a los docentes espacios de capacitación y formación, posibilidad de nuevos aprendizajes, desarrollando un autoaprendizaje que les permitió mejorar la enseñanza; igualmente a los estudiantes, padres/madres de familia y cuidadores, quienes obtuvieron aprendizajes prácticos y significativos desde esta nueva, inesperada y relevante herramienta educativa que dinamizó, enriqueció, fortaleció y diversifico el uso del Internet en los procesos con diversas estrategias pedagógicas como videos, audios, video llamadas, clases grabadas, entre otras.

"Dejarle al estudiante la posibilidad de que él se autoeduque, autoaprendizaje, coloque su ritmo de aprendizaje de enseñanza como él lo considere en casa nos permite que por ejemplo eduquemos niños más despierto más abiertos a las nuevas tendencia que ahoritica se están proyectando para el mundo, que no vamos desfachados de la realidad de nuestro entorno, entonces el Internet ha sido crucial que ha permitido que el estudiante no coma todo lo que le da el docente sino que también busque sus estrategias" (S18. P6).

Y en la Comunicación al igual que en la categoría Influencia del Internet en los Procesos Educativos Inclusivos, el impacto del Internet estuvo dado porque fue la herramienta principal, necesaria, eficaz y efectiva que permitió la Comunicación entre todos los actores de la comunidad educativa.

"Porque de todas formas reitero, si no hubiera Internet en este momento hubiera sido terrible la comunicación con los estudiantes para el desarrollo de las actividades escolares" (S11. P6). 
Por otro lado, desde lo regular, se encontraron elementos que se decidieron resaltar con el objetivo de que sean de utilidad para que le permitan a la I.E. poder fortalecer y mejorar los procesos educativos inclusivos.

"Se evidencia la afectación que tuvieron los estudiantes en sus procesos académicos, fueron muy pocos los que lograron alcanzar los objetivos de aprendizaje para el grado escolar y en gran medida podemos decir que fue la falta de poder contar con el Internet de primera mano para todos los estudiantes sin ninguna excepción y que fuese de buena calidad" (S31. P6).

Finalmente, a partir de lo analizado y expuesto anteriormente se logró identificar el impacto que tiene el Internet en los procesos educativos inclusivos desde la perspectiva del docente de la Institución Educativa Nuestra Señora de La Macarena Meta en el año 2020.

Implementación de Nuevas Acciones desde el Uso de Internet en los Procesos Educativos Inclusivos

Tabla 8. Implementación de nuevas acciones desde el uso de Internet en los procesos educativos inclusivos de la IENSM.

\begin{tabular}{cc} 
CRITERIO DE FAVORECIMIENTO & TOTAL \\
\hline Si & 28 \\
No & 3 \\
\hline
\end{tabular}

Fuente: Elaboración propia de los datos obtenidos en las entrevistas a docentes.

Figura 7. Impacto del Internet en los procesos educativos inclusivos en la IENSM en el año 2020.

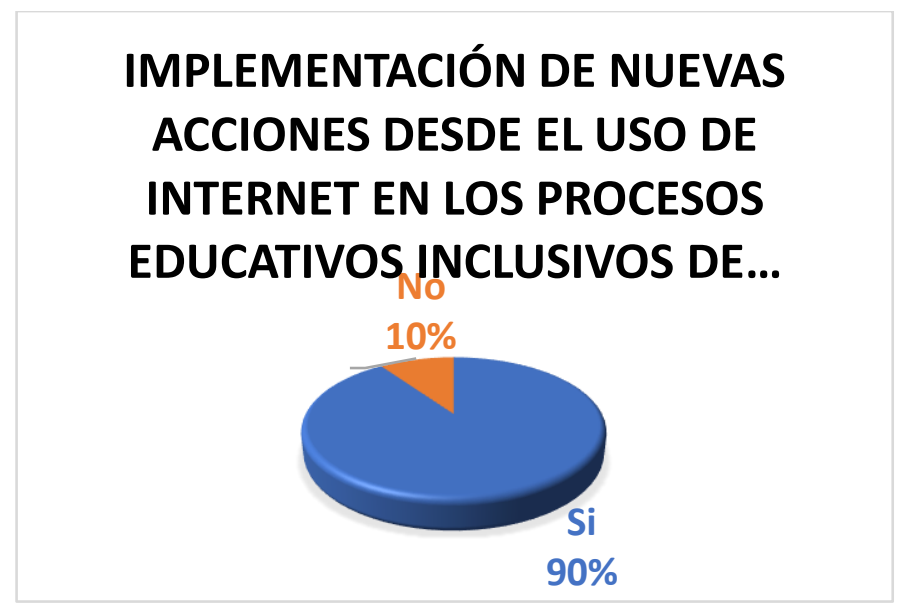

Fuente: Elaboración propia de los datos obtenidos en las entrevistas a docentes.

En la categoría "Implementación de Nuevas Acciones desde el Uso de Internet en los Procesos Educativos Inclusivos" se logró identificar desde la opinión de los docentes cuál fue el criterio de favorecimiento de la implementación de nuevas acciones desde el uso de Internet en los procesos educativos inclusivos de la I.E. Nuestra Señora de La Macarena Meta, mostrados de acuerdo a las respuestas dadas en estos criterios, así: "Si" con un total 
de 28 respuestas representadas en el 90\% y 3 "No" con el 10\%. Es así, como los resultados detallaron en el porcentaje más alto del 90\% que "Si" se deben implementar nuevas acciones desde el uso de Internet, que favorezcan los procesos educativos inclusivos y en un porcentaje menor que "No" con el 16\%.

Analizadas las respuestas afirmativas, se encuentra que la Implementación de Nuevas Acciones desde el Uso de Internet en los Procesos Educativos Inclusivos, parte de las mismas subcategorías mencionadas a lo largo de este análisis, porque esto permite favorecer y mejorar los procesos de Enseñanza y el aprendizaje, adquirir y ofrecerles no sólo a los estudiantes sino también a los docentes Nuevos recursos materiales, tener espacios de Capacitación y formación que permitan la Actualización e innovación en educación de la I.E. para que responda a los cambios, este acorde al momento actual, a las realidades del contexto y necesidades particulares de todos los estudiantes y junto con esto, mejorar la Desigualdad de condiciones existentes en el municipio, que finalmente va a tener como resultado el Mejoramiento continuo, el cual es un proceso dinámico, cambiante, no estático y que tiene como objetivo como su nombre lo indica, mejorar constantemente los procesos a partir de estas nuevas acciones desde el uso de Internet.

"Si contamos con buen Internet y buen acceso a todas estas herramientas tecnológicas no solamente para los niños con discapacidades sino para toda la comunidad educativa en general se van a agilizar los procesos educativos y van a tener como mayor impacto positivo en estos procesos" (S17. P7).

\section{Estrategias Pedagógicas desde el Internet y su Influencia en el Favorecimiento de los Procesos Educativos Inclusivos}

La I.E. Nuestra Señora de La Macarena desde la perspectiva de los docentes, implementó estrategias pedagógicas prácticas y novedosas desde el Internet, utilizando herramientas tecnológicas y virtuales que permitieron y favorecieron atender a los estudiantes de acuerdo a sus necesidades y particularidades y dar continuidad a los procesos educativos inclusivos, donde el Internet fue un elemento esencial y muy importante para la comunicación entre los actores de la comunidad educativa, como Cabero (2001) plantea, las Tecnologías de la Información y la Comunicación (TIC), abarcan y ofrecen diversos medios de interacción, información educativa, mensajería instantánea, audiovisuales, multimedios, Internet, realidad virtual, entre otros, que son utilizados en el ámbito social, para suplir las necesidades de comunicación.

Ahora bien, aunque la I.E. demostró que desarrollaron diferentes estrategias desde el Internet, estas se ven afectadas por la desigualdad de condiciones entre los estudiantes, entre las más relevantes la conectividad que influye en los procesos educativos inclusivos.

\section{Impacto del Internet en la Calidad de los Procesos Educativos Inclusivos}

En síntesis, la I.E. desde la perspectiva de los docentes, tuvo un impacto a través del Internet que le permitió dar continuidad a los procesos educativos inclusivos, buscando garantizar 
así, a pesar de las dificultades presentadas por la emergencia sanitaria del año 2020, el derecho a la educación de los NNAJ, como lo establece la Ley 1098 de 2006 en su Artículo 28 que "los niños, las niñas y los adolescentes tienen derecho a una educación de calidad".

De igual forma, este impacto, se vio reflejado en que la I.E. logró desarrollar estos procesos adecuadamente a partir de diferentes herramientas tecnológicas y virtuales que le permitieron llegar a los estudiantes brindándoles medios como se mencionó anteriormente, pero adicional, espacios de enseñanza y aprendizaje, profundización de conocimientos, de socialización, la posibilidad de nuevos aprendizajes a través de esta herramienta innovadora y práctica que le dio un giro a la educación de los NNAJ y que conllevó a que tanto docentes, estudiantes como padres/madres de familia y cuidadores obtuvieran un aprendizaje práctico y significativo, generando en los estudiantes el desarrollo de autonomía, responsabilidad recíproca y un pensamiento crítico.

Al respecto, Watts y Lee (2017), argumentan que las TIC en la inclusión, favorecen la equidad, pertinencia e inclusión educativa en los entornos de aprendizaje y enseñanza escolar promoviendo espacios para el desarrollo de habilidades como razonar, inferir, interpretar, argumentar, proponer, tomar decisiones, resolver problemas, entre otros, siendo a través de su uso, mediadores del aprendizaje, incluso en personas en situación de discapacidad, lo cual es fundamental para alcanzar el propósito de calidad educativa.

A partir de las cinco dimensiones, las características del educando, los contextos, los aportes materiales y humanos, la enseñanza y el aprendizaje y los resultados, propuestas por la UNESCO (2009), la calidad de los procesos educativos inclusivos de la I.E. reflejó el desarrollo de procesos adecuados, sin embargo, tuvo algunas falencias en las dimensiones mencionadas anteriormente, determinadas principalmente por la ausencia o necesidad de un mayor fortalecimiento en el acompañamiento y apoyo de padres/madres de familia y cuidadores, en los aprendizajes, en la capacitación y formación en el uso del Internet y herramientas tecnológicas para los estudiantes, docentes y padres/madres de familia y cuidadores, en la conectividad, en herramientas y materiales físicos y tecnológicos, en los resultados poco favorables, en capacitación para los docentes para mejorar la atención a todos los estudiantes y en la comunicación.

Por lo que es necesario, que la I.E. supla estas necesidades prioritarias y esté en un mejoramiento constante de sus procesos para que se puedan dar genuinos y efectivos procesos educativos inclusivos de calidad, como el MEN (2013) plantea, en una de las características de la educación inclusiva, la calidad, que según Giraldo et al. (2007), esta permite el desarrollo integral de la persona, la adaptación del conocimiento a las condiciones y necesidades particulares de los estudiantes, además debe estar en los insumos, en la infraestructura, en las técnicas pedagógicas y estrategias metodológicas de enseñanza, en los currículos, en todos los elementos que se utilicen, para que la enseñanza y el aprendizaje se realice con efectividad y así sean reflejados en los resultados personales y académicos de los estudiantes. 
Aunque la I.E. reflejó falencias, también dio a conocer aspectos que fueron significativos en la calidad de los procesos educativos inclusivos, dentro de los más relevantes fueron, que pese a que no todos los estudiantes contaron con el acceso a Internet, tuvieron en cuenta sus necesidades y particularidades que se dieron entorno a la estrategia "Aprender en casa", que a pesar de las dificultades de conectividad y del cambio y reto inesperado en la educación, hicieron uso del Internet para reinventarse, innovar, diseñar e implementar metodologías nuevas, estrategias, actividades, recursos, materiales, brindándoles a los estudiantes diferentes opciones y posibilidades de aprendizaje, las cuales fueron asertivas y contextualizadas, permitiendo resultados satisfactorios, siendo además un gran aporte para los estudiantes, padres/madres de familia, cuidadores y docentes, y claramente, para los procesos educativos inclusivos. Desde esta mirada, se observaron elementos esenciales de una educación inclusiva a partir del Internet, que aportaron y demostraron un impacto efectivo y asertivo en la Calidad de los Procesos Educativos Inclusivos.

Como la UNESCO (2009) precisa, la educación inclusiva, identifica y responde a la diversidad de las necesidades de todos los niños, niñas, adolescentes y jóvenes (NNAJ) a través de una mayor participación en los aprendizajes, las actividades culturales y comunitarias, así como disminuye las brechas en la enseñanza y el aprendizaje, reduciendo la exclusión en la educación. Involucra cambios y modificaciones de contenidos, enfoques, estructuras y estrategias basadas en una visión común y holística que incluye a todos los NNAJ escolares y la convicción sobre la responsabilidad que tiene el sistema educativo en este proceso.

Por todo lo anterior, se destacó el impacto del Internet en la calidad de los procesos educativos inclusivos, siendo el Internet fundamental, como Castells (2015) afirma, el Internet es una de las características más significativas del tiempo, es un importante instrumento para el acceso y proceso de la información, para facilitar el aprendizaje mediante el uso de materiales didácticos interactivos, convirtiéndose en una nueva forma de enseñanza y aprendizaje, sin barreras espacio temporal para el acceso a la información y para la comunicación interpersonal, ofreciendo múltiples posibilidades de innovación educativa en el marco de la enseñanza más personalizada y de un aprendizaje cooperativo acorde con los planteamientos socio constructivistas.

\section{DISCUSIÓN Y CONCLUSIONES}

Si bien en el año 2020, la educación en Colombia y quizás en muchos países por la situación de la pandemia por COVID-19 dio un giro inesperado y complejo para muchos de los actores de la comunidad educativa, a la vez tuvo un cambio novedoso y significativo para la educación y así, para cada uno de sus actores que la involucran, como bien lo demostraron en los resultados obtenidos a través de sus respuestas y narrativas. Los docentes de la I.E. Nuestra Señora de La Macarena Meta, desde su perspectiva se buscó identificar el impacto que tuvo el Internet en los procesos educativos inclusivos en el año 2020, en los cuales se 
observaron elementos esenciales de una educación inclusiva a partir del Internet, que aportaron y demostraron un impacto efectivo y asertivo en la Calidad de los Procesos Educativos Inclusivos. Evidenciados igualmente, en datos estadísticos, con un porcentaje positivo del $52 \%$.

Como la UNESCO (2009) precisa, la educación inclusiva, identifica y responde a la diversidad de las necesidades de todos los niños, niñas, adolescentes y jóvenes (NNAJ) a través de una mayor participación en los aprendizajes, las actividades culturales y comunitarias, así como disminuye las brechas en la enseñanza y el aprendizaje, reduciendo la exclusión en la educación, logrando en últimas acabar con ella. Involucra cambios y modificaciones de contenidos, enfoques, estructuras y estrategias basados en una visión común y holística que incluye a todos los NNAJ escolares y la convicción sobre la responsabilidad que tiene el sistema educativo en este proceso.

Por todo lo anterior, se destacó el impacto del Internet en la calidad de los procesos educativos inclusivos, siendo el Internet fundamental. Como Castells (2015) afirma, el internet es una de las características más significativas del tiempo, es un importante instrumento para el acceso y proceso de la información, para facilitar el aprendizaje mediante el uso de materiales didácticos interactivos, convirtiéndose en una nueva forma de enseñanza y aprendizaje, sin barreras espacio temporal para el acceso a la información y para la comunicación interpersonal, ofreciendo múltiples posibilidades de innovación educativa en el marco de la enseñanza más personalizada y de un aprendizaje cooperativo acorde con los planteamientos socio constructivistas.

Igualmente, en investigaciones previas relacionadas con el objeto de estudio, coincidimos con los resultados que arrojaron la investigación realizada por Mejía y Gómez (2017), acerca del Internet como herramienta didáctica, encontrando que la importancia de contar con Internet en el hogar es prioritaria para el desarrollo de tareas y consulta de la información, dado que aquellos que no cuentan con este tipo de herramientas les impide el acceso a las mismas, planteando que Internet ha dado inicio a desarrollar nuevos modelos y estrategias de aprendizaje, para fortalecer la formación de los estudiantes.

También, concordamos con los resultados de Watts y Lee (2017), que en su investigación acerca de las TIC como herramientas de inclusión educativa, lo cual arrojó como resultados que las posibilidades de las TIC son diversas, pero al mismo tiempo limitadas; debido a que la mayoría de sistemas, no permiten el acceso igual y equitativo para todos, lo que les permitió reconocer que el rol de las TIC en la práctica de la educación inclusiva es fundamental, dado que su utilización, por un lado, favorece el propósito de una educación de calidad, pero además, con su aplicación, se puede eliminar las barreras que impiden el acceso a la formación.

Por lo tanto, se dejan algunas sugerencias de posibles propuestas investigativas que se pueden desarrollar a futuro, con la proyección de que le permitan realizar a las Instituciones Educativas, una mirada analítica, reflexiva, comprensiva, crítica, constructiva y de 
mejoramiento de la calidad de educación brindada a los niños, niñas, adolescentes y jóvenes y que además, puedan fortalecer y enriquecer unos genuinos procesos educativos inclusivos, estas son: Internet como herramienta significativa en la nueva realidad educativa; internet en la educación como herramienta transformadora de la sociedad; ventajas y desventajas de las estrategias pedagógicas implementadas a través de internet en los procesos educativos inclusivos; desventajas que tiene el internet en la educación inclusiva de los niños, niñas y adolescentes; influencia del internet en los procesos de enseñanza de los docentes, influencia del internet en los procesos de aprendizaje de los estudiantes; ventajas y desventajas de la educación por medio del internet en el desarrollo y crecimiento de los niños, niñas y adolescentes y beneficios para los padres/madres de familia y cuidadores del uso del internet en la educación de sus hijos.

Finalmente, de acuerdo al desarrollo de la investigación, se concluyó que el acceso a Internet es una herramienta necesaria para lograr el objetivo en los procesos de educación inclusiva, que según el Ministerio de Tecnologías de la Información y las Comunicaciones (MinTIC, 2020), es el acceso universal a la Web, independientemente del tipo de hardware, software, infraestructura de red, idioma, cultura, localización geográfica y capacidades de los usuarios, por medio del cual les permitió a los estudiantes de la I.E. NSM, buscar y utilizar diferentes formas de acceso a Internet para continuar con su proceso académico desde la estrategia "Aprender en casa". En este sentido, se establecieron las formas de acceso a Internet que tuvieron los estudiantes en el año 2020.

Al examinar la calidad de los procesos educativos inclusivos, la I.E. presentó falencias en las cinco dimensiones planteadas por la UNESCO (2009), determinadas principalmente por la ausencia o necesidad de un mayor fortalecimiento(en algunos de sus elementos) en el acompañamiento y apoyo de padres/madres de familia y cuidadores, en los aprendizajes, en la capacitación y formación en el uso del Internet y herramientas tecnológicas para los estudiantes, docentes y padres/madres de familia y cuidadores, en la conectividad, en herramientas y materiales físicos y tecnológicos, en los resultados poco favorables, en capacitación para los docentes para mejorar la atención a todos los estudiantes y en la comunicación.

Pero a pesar de esto, resaltaron también, aspectos significativos en la calidad de los procesos educativos inclusivos, dentro de los más relevantes fueron, que pese a que no todos los estudiantes contaron con el acceso a Internet, tuvieron en cuenta sus necesidades y particularidades que se dieron entorno a la estrategia "Aprender en casa", que a pesar de las dificultades de conectividad y del cambio y reto inesperado en la educación, hicieron uso del Internet para reinventarse, innovar, diseñar e implementar metodologías nuevas, estrategias, actividades, recursos, materiales, brindándoles a los estudiantes diferentes opciones y posibilidades de aprendizaje, las cuales fueron asertivas y contextualizadas, permitiendo resultados satisfactorios, siendo además un gran aporte para los estudiantes, padres/madres de familia, cuidadores y docentes, y claramente, para los procesos educativos inclusivos. 
Es así como desde la UNESCO (2005), plantea, que el objetivo de la inclusión es brindar respuestas apropiadas al amplio espectro de necesidades de aprendizaje tanto en entornos formales como no formales de la educación. La educación inclusiva, más que un tema marginal que trata sobre cómo integrar a ciertos estudiantes a la enseñanza convencional, representa una perspectiva que debe servir para analizar cómo transformar los sistemas educativos y otros entornos de aprendizaje, con el fin de responder a la diversidad de los estudiantes. El propósito de la educación inclusiva es permitir que los maestros y estudiantes se sientan cómodos ante la diversidad y la perciban no como un problema, sino como un desafío y una oportunidad para enriquecer las formas de enseñar y aprender (p.14).

Al analizar el impacto que tuvo el Internet en los procesos educativos inclusivos desde la perspectiva de los docentes, se encontró que la I.E. pudo a través del Internet dar continuidad a los procesos educativos inclusivos, buscando garantizar así, a pesar de las dificultades presentadas por la emergencia sanitaria del año 2020, el derecho a la educación de los NNAJ, como lo establece la Ley 1098 de 2006 en su Artículo 28 que "Ios niños, las niñas y los adolescentes tienen derecho a una educación de calidad".

De igual forma, este impacto, se vio reflejado en que la I.E. logró desarrollar estos procesos adecuadamente a partir de diferentes herramientas tecnológicas y virtuales que le permitieron llegar a los estudiantes brindándoles medios, pero adicional, espacios de enseñanza y aprendizaje, profundización de conocimientos, de socialización, la posibilidad de nuevos aprendizajes a través de esta herramienta innovadora y práctica que le dio un giro a la educación de los NNAJ y que conllevó a que tanto docentes, estudiantes como padres/madres de familia y cuidadores obtuvieran un aprendizaje práctico y significativo, generando en los estudiantes el desarrollo de autonomía, responsabilidad recíproca y un pensamiento crítico.

Al respecto, Watts y Lee (2017), argumentan que las TIC en la inclusión, favorecen la equidad, pertinencia e inclusión educativa en los entornos de aprendizaje y enseñanza escolar promoviendo espacios para el desarrollo de habilidades como razonar, inferir, interpretar, argumentar, proponer, tomar decisiones, resolver problemas, entre otros, siendo a través de su uso, mediadores del aprendizaje, incluso en personas en situación de discapacidad, lo cual es fundamental para alcanzar el propósito de calidad educativa.

A partir de las experiencias vividas por los docentes en el año 2020 a través del Internet, permitió poder analizar y reflexionar sobre la importancia y necesidad básica y prioritaria, de que el Internet es parte fundamental como herramienta educativa para que las Instituciones Educativas estén acordes al contexto actual de la educación y de los estudiantes.

Con el desarrollo de la investigación, se cumplió con el objetivo propuesto de identificar el impacto que tiene el Internet en los procesos educativos inclusivos desde la perspectiva del docente de la Institución Educativa Nuestra Señora de La Macarena Meta en el año 2020, con el cual fue posible resaltar la importancia y relevancia que tiene el Internet en los 
procesos educativos inclusivos, que aporta, fortalece y por medio de un mejoramiento continuo conlleva a tener una mejor calidad en la educación inclusiva.

Las limitaciones presentadas durante el proceso de investigación giraron en torno a la aplicación del instrumento, dado que aunque la población proyectada en este estudio, era de 54 docentes de aula, no se lograron entrevistar en su totalidad, porque no brindaron espacios para este ejercicio, debido que al realizar su trabajo virtualmente, les ha excedido sus labores dificultando el tiempo para otras actividades, igualmente, hubo docentes que se retiraron de la Institución por razones personales y reubicación y docentes en licencia de maternidad.

\section{REFERENCIAS}

Ainscow, M. \& Echeita, G. (2011). La educación inclusiva como derecho. Marco de referencia y pautas de acción para el desarrollo de una revolución pendiente. $L a$ educación inclusiva. El camino hacia el futuro. 48 ${ }^{a}$ Conferencia Internacional de Educación promovida por la UNESCO y el BIE, Ginebra, Suiza.

Batthyány, K. \& Cabrera, M. (2011). Metodología de la investigación en ciencias sociales. Apuntes para un curso inicial. Montevideo, Uruguay.

Bautista, C. (2011). Proceso de la investigación cualitativa: Epistemología, metodología y aplicaciones. Colombia: El Manual Moderno Colombia.

Castells, M. (2015). MVD en red. El Internet.

Consejo Nacional de Política Económica y Social. (2020). Tecnologías para aprender: Política nacional para impulsar la innovación en las prácticas educativas a través de las tecnologías digitales. Recuperado de https://colaboracion.dnp.gov.co/CDT/Conpes/Econ\%C3\%B3micos/3988.pdf

El Congreso de Colombia. (2006). Ley 1098 de 2006. Recuperado de http://www.secretariasenado.gov.co/senado/basedoc/ley_1098_2006.html

Hernández, R., Fernández, C. \& Baptista, M. (2014). Metodología de la investigación. México D.F.: McGraw-Hill Interamericana.

Hurtado, J. (2000). Metodología de la investigación holística. Caracas, Venezuela: Sypal.

Husserl, E. (1998). Invitación a la fenomenología. Barcelona: Paidós.

Institución Educativa Nuestra Señora de La Macarena. (2018). Proyecto Educativo Institucional P.E./. La Macarena, Colombia. 
Mejía, G. \& Gómez R. (2017). Internet como herramienta didáctica en la formación académica en alumnos de nivel medio superior. Revista Iberoamericana de las Ciencias Sociales y Humanísticas, 6(11).

Ministerio de Salud. (1993). Resolución 008430 de 1993. Recuperado de https://urosario.edu.co/Escuela-Medicina/Investigacion/Documentos-deinteres/Files/resolucion_008430_1993.pdf

Organización de las Naciones Unidas para la Educación, la Ciencia y la Cultura. (2005). Guidelines for inclusión: Ensuring Access to Education for All. Recuperado de http://unesco.org/educacion/inclusive

Organización de las Naciones Unidas para la Educación, la Ciencia y la Cultura. (2009). Directrices sobre políticas de inclusión en la educación. Recuperado de https://unesdoc.unesco.org/ark:/48223/pf0000177849_spa

Watts, C. \& Lee, L. (2017). Las TIC como herramientas de inclusión educativa. Acta Scientiæ Informaticæ, 1(1), 91-97. 